\title{
Hormigones coloreados sustentables: Propiedades y durabilidad
}

\section{Sustainable colored concrete: Properties and durability}

Presentación: 06-07/10/2020

\section{Doctorando:}

\section{Verónica Fernanda Artigas}

Centro de Investigación, Desarrollo y Transferencia de Materiales y Calidad (CINTEMAC) Facultad Regional Córdoba, Universidad Tecnológica Nacional/ Universidad Nacional de Salta - Argentina

veronicaartigas6@gmail.com

\section{Director/a:}

\section{María Josefina Positieri}

\section{Co-director/a:}

\section{María Virginia Quintana}

\section{Resumen}

En este trabajo se buscan probar los beneficios de la incorporación de desechos de perlita cruda finamente molida como reemplazo parcial del cemento Portland en hormigones autocompactantes coloreados (HAC-C). Se plantean distintas dosificaciones con adición de cantidades crecientes de este material en la mezcla con el fin de analizar las propiedades en estado fresco y endurecido de los hormigones. Los resultados permiten definir la viabilidad del uso de este desecho como reemplazo de parte del cemento en los HAC-C.

Palabras clave: HAC-C, Perlita, Sustentabilidad.

\begin{abstract}
This research has the objective to prove the benefits of incorporating finely ground perlite wastes as a replacement for Portland cement in colored self-compacting concretes (C-SCC). Different dosages with increasing amounts of this material are proposed in the mixture in order to analyze concrete fresh and hardened state properties. The results obtained allow to define the feasibility of the use of this waste as a partial replacement of cement in C-SCC.
\end{abstract}

Keywords: C-SCC, Perlite, Sustainability.

\section{Introducción}

La industria del cemento pertenece al grupo de industrias pesadas, es decir, que sus productos son fabricados a gran escala. Para el desarrollo de un país se requieren grandes cantidades de cemento con fines constructivos y, considerando el rápido crecimiento de las ciudades, la necesidad de este producto básico será aún mayor. La creciente demanda de cemento en los últimos años ha llevado al aumento en su producción, observándose una tendencia al alza diario. La producción mundial de cemento es de más de 4 millones de toneladas métricas. Esta creciente demanda, existente y futura, de cemento causa una severa contaminación ambiental de varias maneras. El gran requerimiento de energía y 
materias primas y la emisión de partículas y gases son algunos de los problemas más graves durante la producción de cemento (Nidheesh \& Suresh Kumar, 2019).

Desde el punto de vista de la tecnología de los materiales es importante el desarrollo de hormigones sustentables que tiendan a mitigar los impactos al medio ambiente generados por la construcción.

Durante la historia del hormigón y con distintos objetivos se han utilizado las adiciones minerales activas, cuyas funciones cubre actualmente aspectos de índole económica, ecológica y tecnológica. Económica, porque disminuyen el contenido de cemento en el hormigón, el cual consume gran cantidad de energía para su producción. Ecológica, porque al reemplazar parte del cemento se disminuye la emisión de $\mathrm{CO}_{2}$, se optimiza la utilización de recursos no renovables que se emplean en la fabricación del mismo y además el hormigón admite, en muchos casos, subproductos o desechos industriales como materiales componentes. Y la tecnológica, porque según sus interacciones con el sistema pasta cementicea-agregado contribuyen al mejoramiento de algunas propiedades del hormigón (Rahhal \& Eperjeci, 2012; Raggiotti, 2015).

La sustitución de clinker por otros materiales es una de las formas efectivas de reducir la emisión de dióxido de carbono en la industria del cemento (Garcia-Gusano, Cabal, \& Lechón, 2015).

Actualmente el cuidado del medio ambiente y la reducción de costos de fabricación son importantes temas de discusión a nivel de las industrias. Dentro de esta temática, la industria del cemento ha presentado numerosos cambios, entre ellos, impulsa el uso de materiales naturales, residuales o subproductos industriales en su proceso de fabricación (Fernandez \& Trezza, 2007).

La perlita es una roca de origen volcánico, cuya propiedad sobresaliente es de expandirse cuando es sometida a la acción del calor. Expandida, es utilizada principalmente en la industria de la construcción como aislante térmico y acústico. Debido a su estructura vítrea y a los altos contenidos de $\mathrm{SiO}_{2}$ y $\mathrm{Al}_{2} \mathrm{O}_{3}$, la perlita es una puzolana. Sin embargo, sus características puzolánicas se han mencionado solo en un número limitado de documentos técnicos (Pachta, Papadopoulos, \& Stefanidou, 2019; Abdulkader \& Salem, 2017; Erdem, Meral, Tokyay, \& Erdogan, 2007; Yu, Ou, \& Lee, 2003; Demirboga, Örüng, \& Gül, 2001; Erdem E. , 1997; Urhan, 1987).

Este vidrio natural de origen volcánico es extraído por métodos a cielo abierto en distintas partes del mundo. En Argentina existen yacimientos de perlita en la zona de la Puna. En San Antonio de los Cobres (Salta) se sitúan numerosas canteras de extracción de perlita, la misma es sometida a un proceso mecánico para llevar la roca a un tamaño más pequeño y así poder transportarlo a la planta procesadora, generándose desechos finos del material.

La incorporación de los desechos de perlita cruda finamente molidos (FP) como reemplazo parcial del cemento en los HAC-C, temática que aborda este trabajo, contribuirá al estudio y análisis de viabilidad del uso de este material en el ámbito de la construcción.

\section{Desarrollo}

El primer paso implica la caracterización de los materiales a utilizar:

- Cemento: Se utilizó cemento Pórtland CPC40 de procedencia local, el cual fue caracterizado químicamente y se determinó su densidad (IRAM 1624) y superficie específica (IRAM 1623).

- Finos de perlita: Se realizó la determinación de densidad, pasante del tamiz № 200, superficie especifica (IRAM 1623), composición química, índice de actividad puzolánica con cemento portland y con cal y requerimiento de agua (IRAM 1654), método de ensayo de la puzolanicidad para los cementos puzolánicos (IRAM 1651), contracción por secado (IRAM 1761). 
- Pigmentos: Se utilizó pigmentos inorgánicos a base de óxidos de hierro, de dos colores distintos. Se determinó su densidad, superficie específica (Blaine) y composición química. Ensayos según ASTM C 979.

- Agregados finos: Granulometría (IRAM 1505), módulo de finura (IRAM 1505), densidad condición saturada a superficie seca (IRAM 1520) y absorción (IRAM 1520). Agregados gruesos: Granulometría (IRAM 1505), módulo de finura (IRAM 1505), densidad (IRAM 1533), P.U.S. (IRAM 1548), P.U.C. (IRAM 1548), contenido de espacios vacíos (IRAM 1548) y absorción (IRAM 1533).

- Superfluidificante: Se determinó el residuo sólido.

Se continúa con el análisis de compatibilidad de cementos y aditivos, para lo cual se elaboran y ensayan pastas cementiceas:

- Dosificación de pastas con distintos porcentajes de FP, variando la relación perlita/cemento en 0,10; 0,15 y 0,20. La relación agua/cemento se mantuvo fija en 0,4. En cuanto al porcentaje de pigmento, se adoptó un porcentaje del $6 \%$ en peso del cemento, ya que de acuerdo a la bibliografía es un porcentaje a partir del cual un incremento de la cantidad de pigmento no genera cambios significativos en el color. La cantidad de aditivo se fija como porcentaje del contenido de cemento en la mezcla, para cada una de las pastas se varió el porcentaje de aditivo superfluidificante hasta alcanzar la dosis óptima. En la Figura 1 se presentan las composiciones de cada una de las pastas elaboradas.

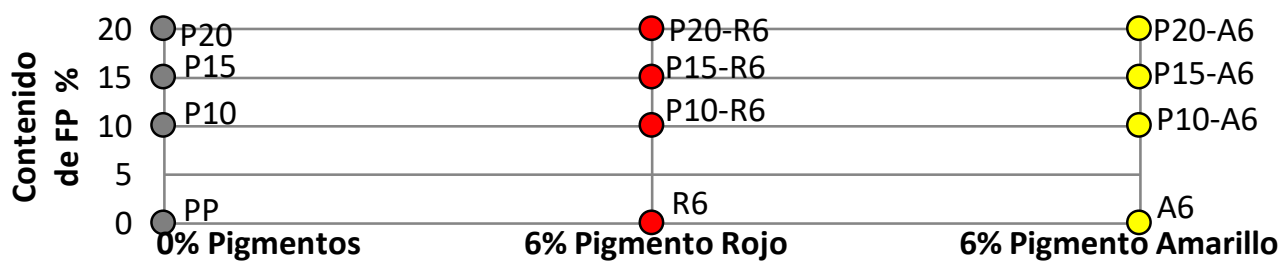

Figura 1: Dosificación de pastas

- Cada una de las pastas elaboradas fue ensayada para determinar el porcentaje de aditivo correspondiente a la dosis óptima mediante los ensayos de cono de Marsh y de Mini-Slump, realizados a los 5, 30, 60 y 90 minutos desde el momento en que toman contacto el cemento y el agua.

Seguidamente se elaboran los HAC-C:

- Dosificación de los hormigones: Se planteó la elaboración de doce familias de hormigones, considerando un hormigón patrón, tres hormigones con contenido creciente de finos de perlita (10, 20 y 30\% en reemplazo del cemento), un hormigón con pigmento amarillo únicamente, otro con pigmento rojo solamente y seis hormigones donde se combinaron los pigmentos y los FP. En todos los casos se mantuvo constante la relación agua/material fino en 0,45, el porcentaje de pasta en 50\% y el porcentaje de agregado grueso en 35\%. La dosis de aditivo superfluidificante fue la necesaria para alcanzar la fluidez buscada en cada caso. La Figura 2 muestra la denominación de cada pastón y su composición.

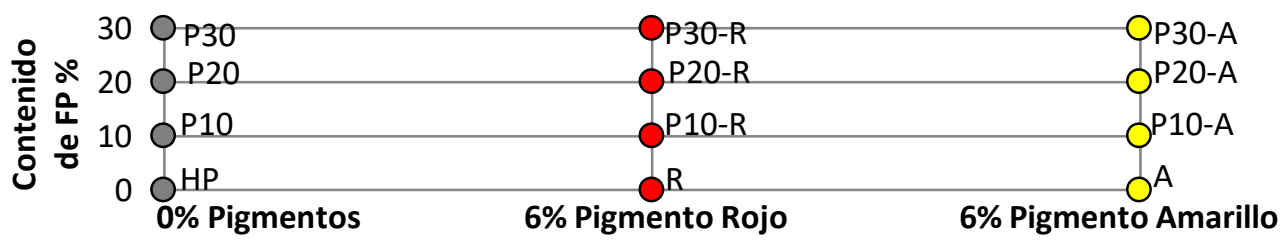

Figura 2: Dosificación de hormigones

- Ensayos sobre los hormigones en estado fresco: Densidad y contenido de aire del Hormigón Fresco (IRAM1562), extendido o asentamiento (según corresponda), extendido con Anillo Japonés, caja L y embudo en V. 
- Ensayos sobre los hormigones en estado endurecido: Medición y evaluación del color a 28, 90 y 180 días, resistencia a compresión a 7, 28, 90 y 180 días (IRAM 1546), módulo de elasticidad a 28, 90 y 180 días (ASTM C 469), capacidad de succión capilar y velocidad de succión capilar a 28 días (IRAM 1871), penetración del agua a presión a 28 días (IRAM 1554), conductividad térmica a 28 días, ultrasonido a 28 días, absorción total a 360 días (ASTM C642), abrasión a 360 días, carbonatación a 720 días, contracción por secado (IRAM 1597).

\section{Resultados}

Caracterización de materiales:

En cuanto a la caracterización de los materiales utilizados, solamente mencionaremos los resultados obtenidos en los ensayos realizados sobre los finos de perlita (FP), ya que los ensayos realizados sobre el cemento, los pigmentos y los agregados son rutinarios y los resultados obtenidos estuvieron siempre dentro de los límites esperados. En la Tabla 1 se presentan los resultados de los ensayos de caracterización realizados sobre los FP.

\begin{tabular}{c|c|c|c|c|c} 
Densidad & $\begin{array}{c}\text { Superficie } \\
\text { específica }\end{array}$ & $\begin{array}{c}\text { Requerimiento de } \\
\text { agua }\end{array}$ & $\begin{array}{c}\text { Contracción por } \\
\text { secado }\end{array}$ & \multicolumn{2}{|c}{$\begin{array}{c}\text { Coeficiente de puzolanicidad } \\
\text { (Frattini) }\end{array}$} \\
\hline 2.23 & $52.8 \mathrm{~m}^{2} / \mathrm{g}$ & $95.4 \%$ & $-0.077 \%$ & 1.17 a 8 días & 1.05 a 15 días
\end{tabular}

Tabla 1: Caracterización de los FP

\section{Ensayos sobre pastas}

De acuerdo a los resultados de los ensayos de cono de Marsh y mini-slump se determinó la dosis de saturación de aditivo superfluidificante para cada una de las pastas en estudio, dichos valores se presentan en la Tabla 2. Con el paso del tiempo los valores de $\mathrm{T}_{115} \mathrm{y}$ de tiempo de paso por el cono de Marsh aumentan y los de diámetro de extendido disminuyen como es lógico debido a la disminución de la fluidez en la mezcla, sin embargo cabe mencionar que tanto la pasta patrón como las pastas con adición de FP y de pigmentos presentan porcentajes similares de variación de valores entre los obtenidos inicialmente y los obtenidos a los 30, 60 y 90 minutos.

\begin{tabular}{c|c|c|c|c|c|c|c|c|c|c|c|c} 
Pasta & $\mathrm{PP}$ & $\mathrm{P} 10$ & $\mathrm{P} 15$ & $\mathrm{P} 20$ & $\mathrm{~A} 6$ & $\mathrm{R} 6$ & $\mathrm{P} 10-\mathrm{A} 6$ & $\mathrm{P} 15-\mathrm{A} 6$ & $\mathrm{P} 20-\mathrm{A6}$ & $\mathrm{P} 10-\mathrm{R} 6$ & $\mathrm{P} 15-\mathrm{R} 6$ & $\mathrm{P} 20-\mathrm{R} 6$ \\
\hline $\begin{array}{c}\text { Dosis óptima } \\
\%\end{array}$ & 0,55 & 0,60 & 0,65 & 0,70 & 0,65 & 0,70 & 0,85 & 1,00 & 1,15 & 0,75 & 0,90 & 0,90
\end{tabular}

Tabla 2: Dosis óptimas de aditivo superfluidificante para cada pasta

\section{Ensayos sobre hormigones}

A continuación se presentan los resultados obtenidos en estado fresco para los hormigones elaborados. La Figura 3 muestra los resultados de diámetro de extendido obtenido con y sin anillo japonés y los valores de T50. En la Figura 4 presenta la relación $\mathrm{H}_{2} / \mathrm{H}_{1}$ del ensayo de la caja en L. La Figura 5 muestra los tiempos de pasaje por el embudo en V.

En cuanto a los resultados en estado endurecido, se tienen hasta el momento los de resistencia a compresión a los 28 y 90 días que se muestran a continuación (Figura 6) Los resultados de las series P30, P30R y P30A y los correspondientes a las edades de 180 se encuentran en proceso. 


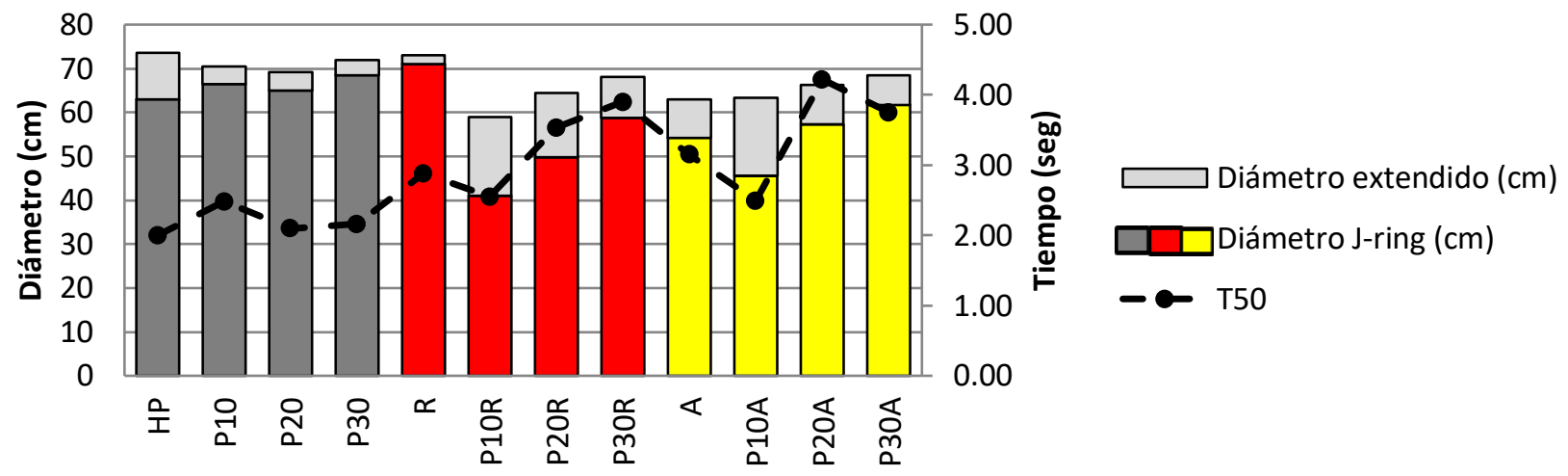

Figura 3: Resultados ensayo de extendido y J-ring

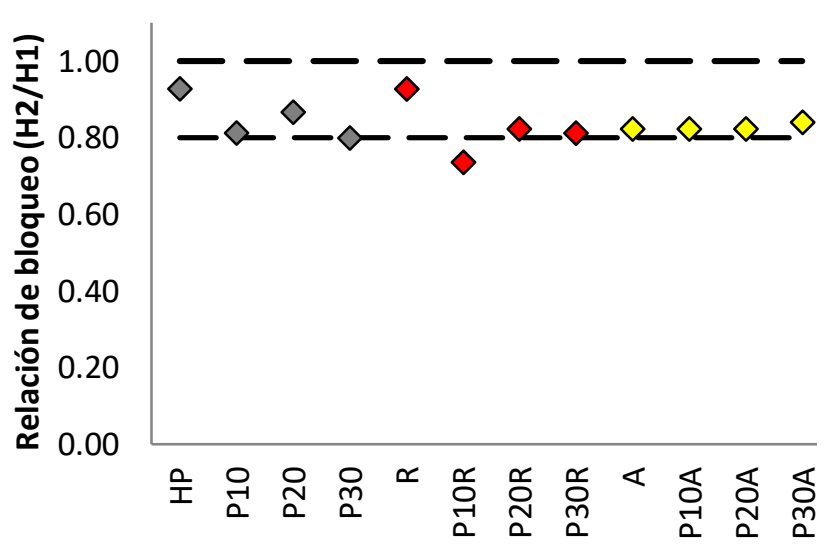

Figura 4: Resultados ensayo caja en L

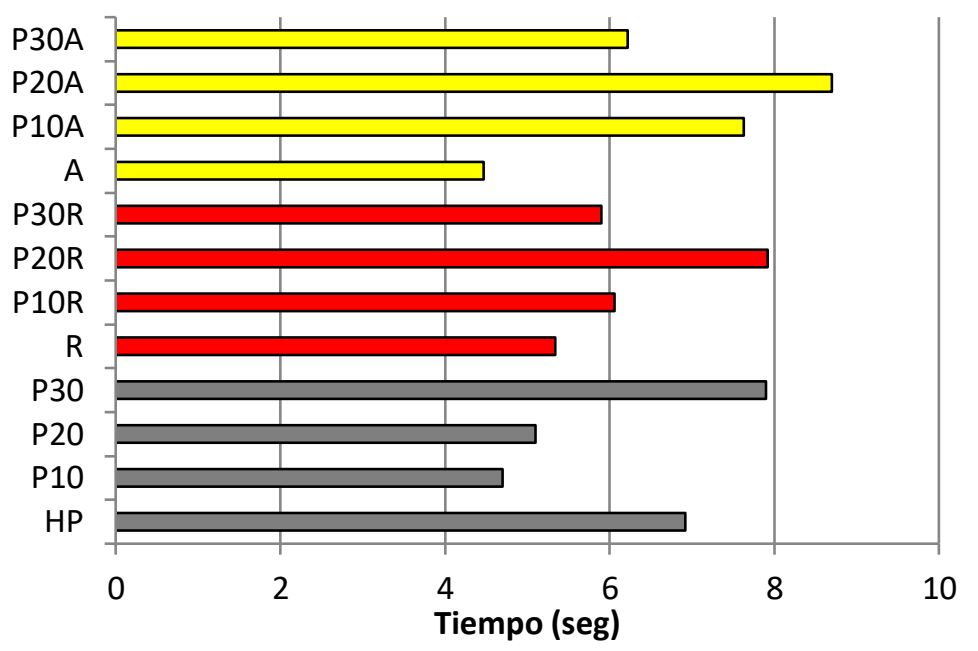

Figura 5: Resultados ensayo embudo en $\mathrm{V}$

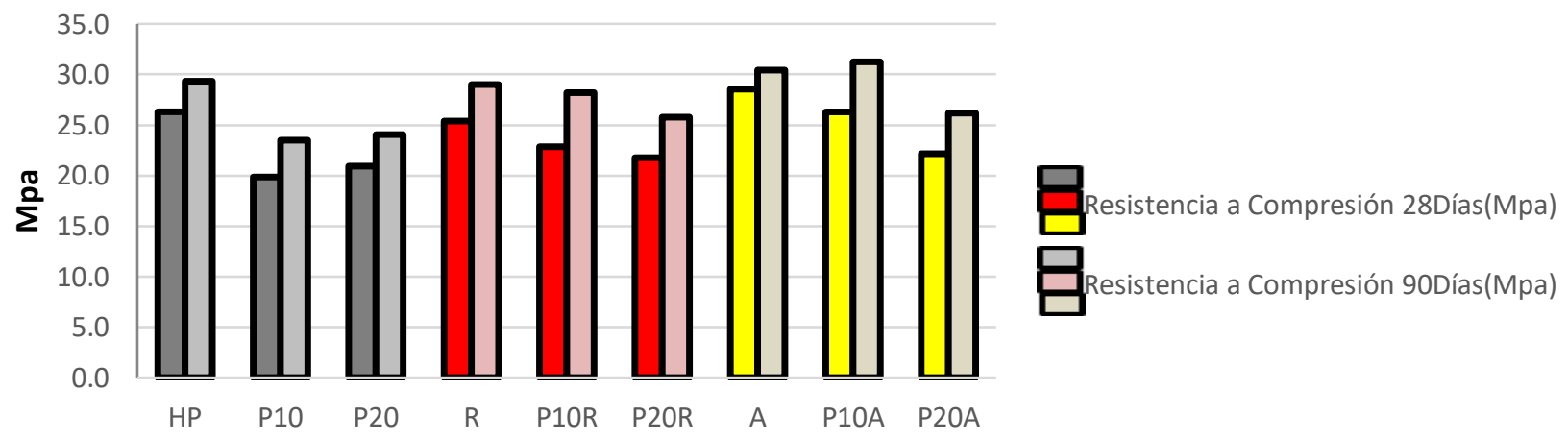

Figura 6: Resultados ensayo resistencia a compresión

\section{Conclusiones}

En cuanto a los ensayos realizados sobre el material FP, se determinó que químicamente el índice de puzolanicidad obtenido a la edad de 15 días no muestra actividad puzolanica, por lo que se están realizando los ensayos físicos correspondientes para la determinación del índice de puzolanicidad del material con cemento Portland y con cal. 
Los ensayos realizados sobre las pastas cementiceas y los hormigones muestran que la incorporación de FP a la mezcla produce un aumento de la cohesión y una disminución en la fluidez debida a la absorción de agua por parte de los FP, lo que se traduce en la necesidad de una mayor dosis de aditivo superfluidificante para alcanzar la saturación. Paralelamente, la adición de pigmento amarillo también produjo disminución de la fluidez de la mezcla, debido a la forma alargada de sus partículas.

La mayoría de los hormigones elaborados incorporando distintas combinaciones de FP y pigmentos cumplen con los parámetros de autocompactabilidad mencionados en la bibliografía para los distintos ensayos en estado fresco.

La incorporación de FP produce una marcada disminución de la resistencia a compresión en los hormigones a la edad de 28 días, siendo que a los 90 días los resultados tienden a igualarse con el patrón. Los ensayos a 180 días confirmaran si se mantiene esta tendencia. Por otro lado se observa que la forma de aguja de las partículas de pigmento amarillo genera mayor trabazón, aumentando la resistencia del hormigón.

Si bien es necesaria la realización de más ensayos para determinar los efectos de los FP en los hormigones, este trabajo permite vislumbrar las aptitudes de este material para ser considerado en la elaboración de HAC-C, resultando factible la incorporación de hasta el 20\% de FP en la mezcla. Los avances en el conocimiento que tiendan a analizar la factibilidad de la utilización de desechos resultan beneficiosos para el desarrollo sustentable.

\section{Referencias}

Abdulkader, E. M., \& Salem, G. N. (2017). Utilization of industrial waste perlite powder in self-compacting concrete. Journal of Cleaner Production 156, 507-517.

Demirboga, R., Örüng, I., \& Gül, R. (2001). Effects of expanded perlite aggregate and mineral admixtures on the compressive strenght of low-density concretes. Cem. Concr. Res. 31 (11) , 1627-32.

Erdem, E. (1997). Efect of various additives on the hydration of perlite-gyspum plaster and perlite-Portland cement pastes. Turk J Chem 21 (3) , 209-214.

Erdem, T. K., Meral, Ç., Tokyay, M., \& Erdogan, T. Y. (2007). Use of perlite as a puzzolanic addition in producin blended cements. Cement \& Concrete Composites $29,13-21$.

Fernandez, L. I., \& Trezza, M. A. (2007). Estudio de la aptitud del loess pampeano como adición activa al cemento Portland. Revista de la Contrucción 6 (1) , 4-12.

Garcia-Gusano, D., Cabal, H., \& Lechón, Y. (2015). Long-term behavior of CO2 emissions from cement production in Spain: Scenario analysis using an energy optimisation model. J. Clean. Pro. 99, 101-111.

Nidheesh, P. V., \& Suresh Kumar, M. (2019). An overview of environmental sustainability in cement and steel production. Journal of Clenner Production 231, 856-871.

Pachta, V., Papadopoulos, F., \& Stefanidou, M. (2019). Development and testing of grouts based on perlite by-products and lime. Construction and Building Materials 207 , 338-344.

Raggiotti, B. (2015). Hormigones con adiciones activas: Diseño, optimización y caracterización con criterio de sustentabilidad. Tesis doctoral.

Rahhal, V., \& Eperjeci, L. (2012). Cap 3: Adiciones minerales. En Ese material llamado Hormigón (págs. 79-92). AATH.

Urhan, S. (1987). Alkali silica and pozzolanic reactions in concrete. Part II: Observation on expanded perlite aggregate concretes. Cem. Concr. Res. 17 (3) , 209-214.

Yu, L.-H., Ou, H., \& Lee, L.-L. (2003). Investigation on puzzolanic effect of perlite powder in concrete. Cem. Concr. Res. 33 (1), 73-76. 